\title{
Cooxidation not to be Confused with Catalysis: A Chemical Education Article to Physical-organic Chemists
}

\author{
R. SANJEEV 1 , D. A. PADMAVATHI ${ }^{2}$ and V. JAGANNADHAM ${ }^{2 *}$ \\ 'Department of Chemistry, Geethanjali College of Engineering and Technology, \\ Cheeryal-501301, India \\ ${ }^{2}$ Department of Chemistry, Osmania University, Hyderabad 500007, India. \\ ${ }^{*}$ Corresponding athor E-mail: jagannadham1950@yahoo.com
}

http://dx.doi.org/10.13005/ojc/340159

(Received: June 24, 2017; Accepted: December 10, 2017)

\begin{abstract}
Two substrates (A) and (B) are oxidized separately by an oxidant (Oxi) with the rate constants $k_{1}$ and $k_{2}$ and they are oxidized taken together $(\mathrm{A}+\mathrm{B})$ under similar conditions with a rate constant $k_{3}$, if the value of $k_{3}=\left(k_{1}+k_{2}\right)$, then it is said to be an example of two reactions "going parallel". If the value of $k_{3} \gg>>\left(k_{1}+k_{2}\right)$, then the redox process is termed as "co-oxidation" (Hasan and Rocek 1972, JACS). In this process in the mixture the two substrates are oxidized synchronously by a direct three electron transfer route if the oxidant happens to be $\mathrm{Cr}(\mathrm{VI})$ and by a direct four electron transfer route if the oxidant happens to be $\mathrm{Mn}(\mathrm{VII})$ (Jagannadham et al., 1986, Oxidation Communications). It was realized that the essential condition of the synchronous oxidation of two substrates $A$ and $B$ is that one substrate must have two functional groups and the other must have one functional group or vice-versa. The compound with two functional groups must be a good chelating agent with the metal ion oxidant. A substrate $(A)$ is oxidized by an oxidant (Oxi) with a rate a constant $k_{4}$ and is oxidized in presence of a catalyst (Cat) with a rate constant $k_{5}$, if $k_{5}>k_{4}$ the redox process is termed as "catalyzed process". It is to be noted that in the catalytic process the catalyst (Cat) is not oxidized and its concentration does not change during the reaction. It only increases the rate of oxidation with lower activation energies. If $k_{5}=k_{4}$ it is to be understood that there is "no catalysis". If $k_{5}<k_{4}$ it is to be understood that the catalyst is called a negative catalyst or "inhibitor" and the reaction goes with higher activation energy. In this paper a lucid description is given for the two processes "co-oxidation" and "catalysis" with putative examples.
\end{abstract}

Keyword: Cooxidation,Catalysis, Chemical education.

\section{INTRODUCTION}

Hasan and Rocek were the first to report a direct synchronous three electron oxidation process where in isopropyl alcohol and oxalic acids were oxidized ${ }^{1}$. Later several publications appeared from his laboratory ${ }^{2-18}$. Sequel to Rocek's discovery ${ }^{1}$ of one step three electron oxidations several

This is an $\mathbf{C}$ Open Access article licensed under a Creative Commons Attribution-NonCommercial-ShareAlike 4.0 International License (https://creativecommons.org/licenses/by-nc-sa/4.0/ ), which permits unrestricted NonCommercial use, distribution and reproduction in any medium, provided the original work is properly cited. 
researchers reported numerous studies in this area. Quoting them in this article is beyond the scope and limit of this article. However to one of the authors of this article the situation warrants to quote our own examples of oxidation of aliphatic esters in presence of oxalic acid again by a synchronous three electron transfer route ${ }^{19}$, and oxidation of isopropanol and lactic acid by $\mathrm{Cr}(\mathrm{VI})^{20}$ and a mixed substrate system of an aldehyde and an alcohol ${ }^{21}$. Another interesting study by Roèek on three electron oxidation is the intra-molecular cooxidation of a single substrate having three functional groups such as 2,7-dihydroxyheptanoic acid by chromic acid $^{13}$. 2,7-dihydroxyheptanoic acid is a substitute of two substrates one with two functional groups and the other with one functional group. Though not effective as 2,7-dihydroxyheptanoic acid oxidation, a similar study was also reported from our laboratory where in oxidation of glycerol which has three hydroxyl groups by chromic acid did take place by a three electron transfer route ${ }^{22}$. Our study was further extended to permanganate ion oxidations where in iso-propanol was oxidized by permanganate ion in presence of several bifunctional compounds by synchronous four electron transfer route ${ }^{23}$.

The end product of the oxidant in three electron oxidations by $\mathrm{Cr}(\mathrm{VI})$ is $\mathrm{Cr}(\mathrm{III})$. As $\mathrm{Cr}(\mathrm{III})$ is stable it does not undergo any further reduction. But the situation in the four electron transfer reaction by $\mathrm{Mn}(\mathrm{VII})$ is different. The end product of $\mathrm{Mn}(\mathrm{VII})$ is $\mathrm{Mn}$ (III) which is known to be reasonably a good oxidant ending with the formation of $\mathrm{Mn}$ (II) as the stable end product ${ }^{24}$. In the previous work ${ }^{23}$ from our laboratory care is taken so that the similar conditions were adopted where in the oxidation of either iso-propanol or the bifunctional compounds by $\mathrm{Mn}(\mathrm{III})$ was negligible.
Catalysis is the process that increases the rate of a chemical reaction due to the participation of a third substance called a catalyst. The catalyst is not consumed in the course of the catalyzed reaction and can continue in several cycles until the completion of the reaction. Very often only micromolar quantities of the catalyst are required in principle ${ }^{25}$. In catalytic process the catalyst is not oxidized or reduced. Hence the process of co-oxidation should not be confused with catalysis.

\section{DISCUSSION}

Before understanding the difference between cooxidation and catalysis it is noteworthy to discuss the oxidations of related compounds by $\mathrm{Cr}(\mathrm{VI})$ and $\mathrm{Mn}(\mathrm{VII})$ in detail.

\section{Oxidation of iso-propanol by $\mathrm{Cr}(\mathrm{VI})$}

Rocek and Krupicka ${ }^{26 a}$ and Wiberg and Schaefer ${ }^{26 b}$ reported the oxidation of iso-propanol by chromic acid. The reaction was shown to undergo via a chromate ester which is formed in a reversible step and then decomposes in a rate determining step to give acetone as shown in the following mechanism (Scheme 1).

$\mathrm{H}^{+}+\mathrm{HCrO}_{4}{ }^{-} \rightleftharpoons \mathrm{H}_{2} \mathrm{CrO}_{4} \ldots$...fast step

$\left(\mathrm{CH}_{3}\right)_{2} \mathrm{CH}-\mathrm{OH}+\mathrm{H}_{2} \mathrm{CrO}_{4} \rightleftharpoons\left(\mathrm{CH}_{3}\right)_{2} \mathrm{CH}-\mathrm{O}-\mathrm{CrO}_{3} \mathrm{H}$ fast step

$\left(\mathrm{CH}_{3}\right)_{2} \mathrm{CH}-\mathrm{O}-\mathrm{CrO}_{3} \mathrm{H} \rightarrow\left(\mathrm{CH}_{3}\right)_{2} \mathrm{C}=\mathrm{O}+\mathrm{H}_{3} \mathrm{O}++\mathrm{HCr}(\mathrm{IV}) \mathrm{O}_{3}-$ slow step

\section{Scheme 1}

\section{Oxidation of oxalic acid by $\mathrm{Cr}(\mathrm{VI})$}

Oxidation of oxalic acid was studied by Bakore and Jain ${ }^{27}$ and later by Hasan and Rocek ${ }^{28}$. The reaction involves a cyclic transition state between chromic acid and oxalic acid which later decomposes in a rate determining step to give $\mathrm{CO}_{2}$ as the end product. The mechanism is shown in Scheme 2.

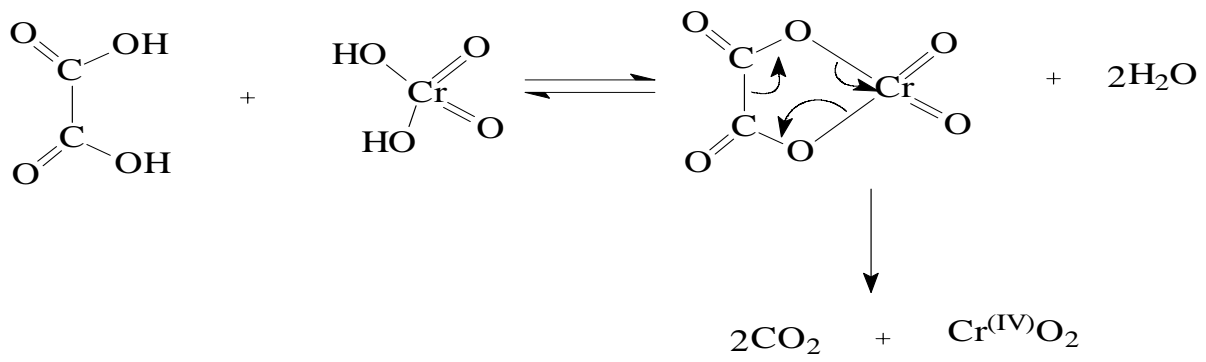

Scheme 2 


\section{Oxidation of a mixture of iso-propanol and oxalic acid by $\mathrm{Cr}(\mathrm{VI})$}

Hasan and Rocek for the first time reported a direct synchronous three electron oxidation process where in isopropyl alcohol and oxalic acids were both oxidized ${ }^{1}$. They strongly advocated the reaction to be a direct three electron transfer route based on the increase in the rate of oxidation by several orders of magnitude compared to the rates of oxidation of the two substrates taken alone. Further their studies were supported by the presence of free radicals and stiochiometry of the reaction. In the mixture oxalic acid was oxidized by one electron transfer and iso-propanol by two electron transfer process. The end products are acetone and $\mathrm{CO}_{2}$. The brief mechanism where in a ter-molecular complex formed was supposed to give products in a slow step as shown in scheme 3 .
Oxidations of a substrate with three functional groups

Another interesting study by Rocek on three electron oxidation is the intra-molecular cooxidation of a single substrate having three functional groups such as 2,7-dihydroxyheptanoic acid by chromic acid ${ }^{13}$. This was successfully discovered from the study of a series of dihydroxy carboxylic acids $\left(\mathrm{HO}\left(\mathrm{CH}_{2}\right)_{n} \mathrm{CHOHCO}_{2} \mathrm{H}\right)$. With $\mathrm{n}=5$ that is 2,7-dihydroxyheptanoic acid reacted completely in a different way compared to the other members of the series. 2,7-dihydroxyheptanoic acid is a substitute of two substrates one with two functional groups and the other with one functional group. The end products were corresponding aldehyde and alcohol. The transition state that formed is shown in scheme 4 .

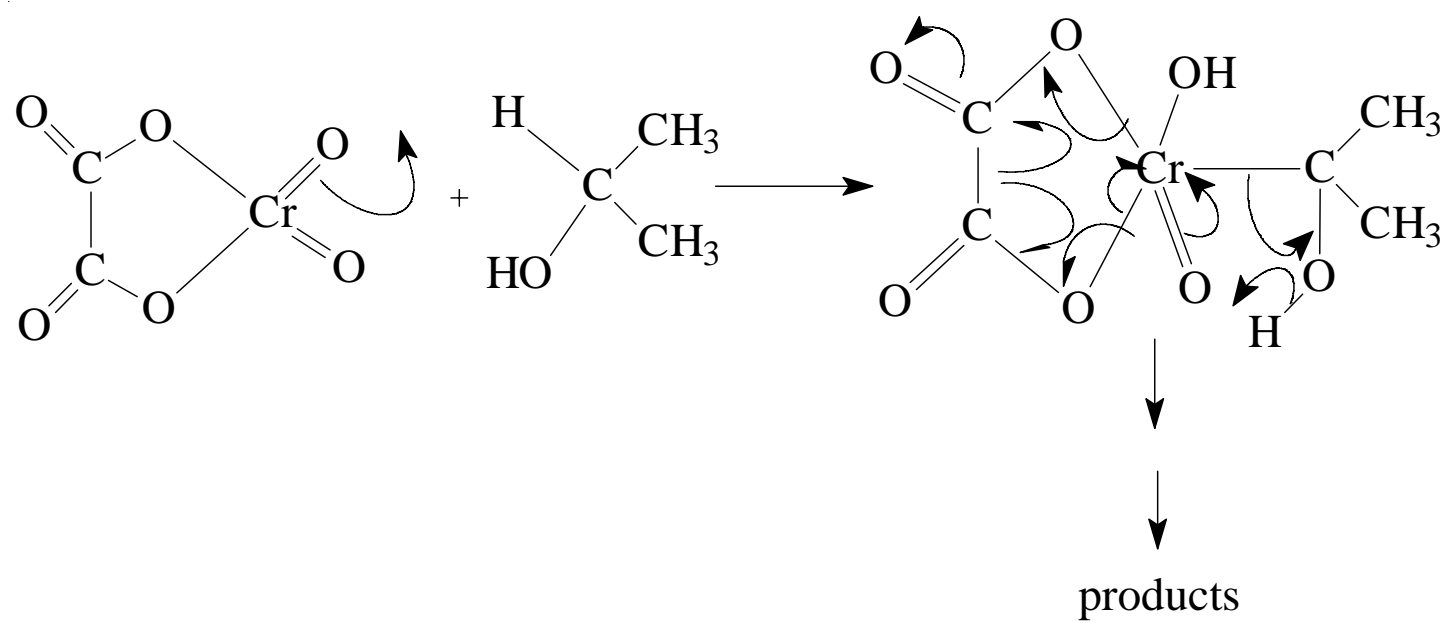

Scheme 3<smiles></smiles> 
Though the reaction sequence is not as efficient as the 2,7-dihydroxyheptanoic acid oxidation by $\mathrm{Cr}(\mathrm{VI})$, a similar study was also reported from our laboratory where in oxidation of glycerol which has three hydroxyl groups by chromic acid did take place by a three electron transfer route 22 . The end product of the reaction was glyoxal. The transition state is shown in scheme 5 .

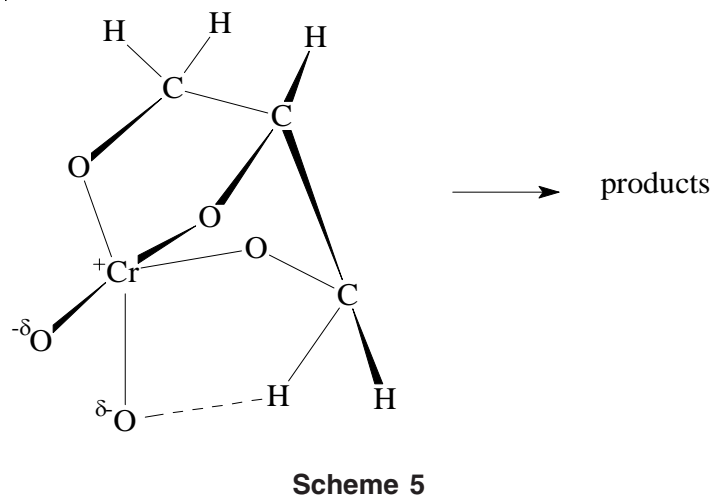

\section{Oxidation of iso-propanol by $\mathrm{Mn}(\mathrm{VII})$}

Kinetic study of oxidation of iso-propanol is more than a century old ${ }^{29}$. The reaction sequence involves the formation of permanganate ester which later gives the acetone as the product in a slow step. The reaction is shown in scheme 6 .

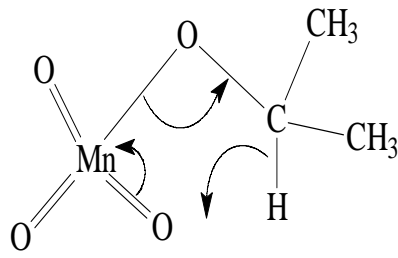<smiles>C=C[As][AsH3]</smiles>

\section{Oxidation of lactic acid by $\mathrm{Mn}(\mathrm{VII})$}

Lactic acid is determined by the reaction of $\mathrm{Mn}(\mathrm{VII})$ in acid medium ${ }^{30}$. The products of oxidation were acetaldehyde and $\mathrm{CO}_{2}$. The reaction sequence is shown in scheme 7 .

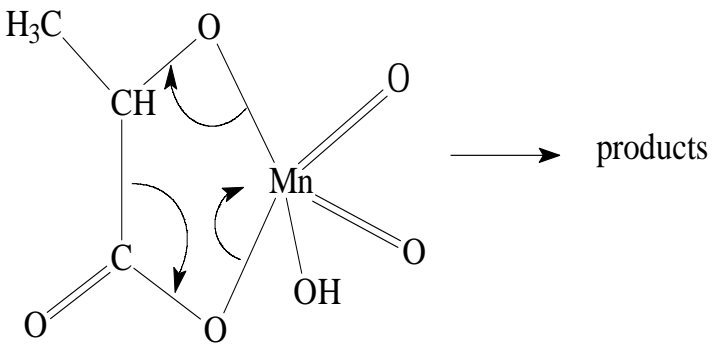

Scheme 7

\section{Cooxidation by Mn(VII)}

Our study was further extended to permanganate ion oxidations where in iso-propanol was oxidized by permanganate ion in presence of several bi-functional compounds by synchronous four electron transfer route ${ }^{23}$. The transition state taking lactic acid and iso-propanol as example is shown in scheme 8.

\section{Catalyzed and un-catalyzed reactions}

There were several thousands of catalyzed reactions reported in literature. Quoting them in the present article is beyond the scope of this article. But as an example to understand the concept of catalysis we present here a study involving $\mathrm{Ag}(\mathrm{l})$ catalyzed and un-catalyzed oxidation of dimethyl sulfoxide (DMSO) by $\mathrm{Ce}(\mathrm{IV})$ in nitric acid medium published from our labratory ${ }^{31}$. The product of oxidation was found to be dimethyl sulfone $\left(\mathrm{DMSO}_{2}\right)$. The mechanism proposed for both the processes were shown in scheme 9.

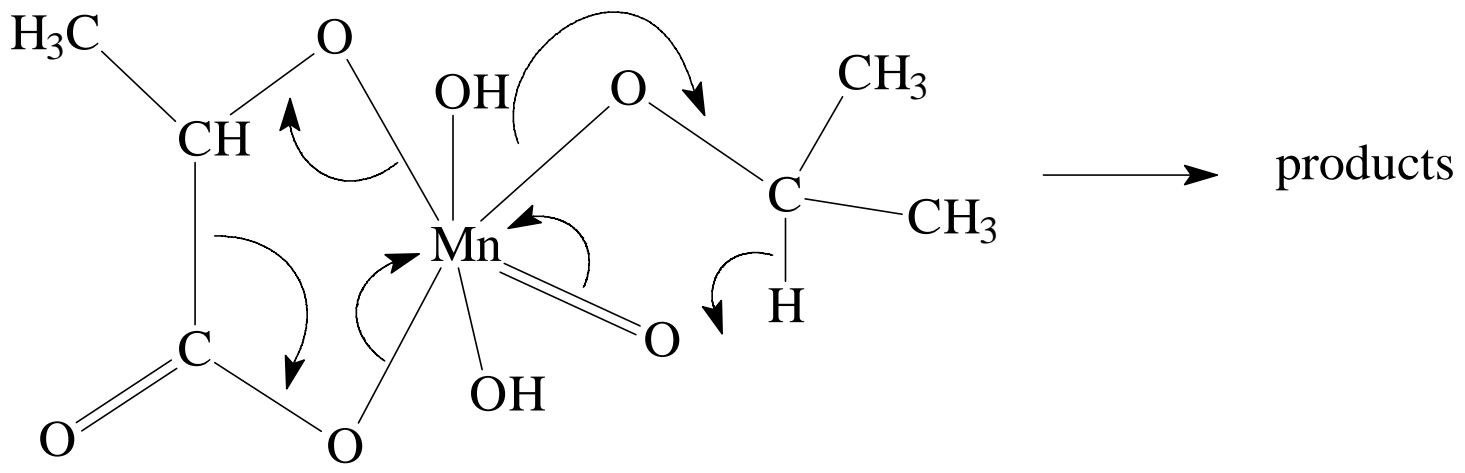

Scheme 8 


\section{Un-catalyzed path}

$\begin{array}{llll}\text { DMSO }+\mathrm{Ce}(\mathrm{IV}) & \rightleftharpoons & \text { complex } & \text { fast step } \\ \text { Complex } & \rightarrow & \mathrm{DMSO}^{\circ+} & \text { slow step } \\ \mathrm{DMSO}^{\circ+}+\mathrm{Ce}(\mathrm{IV})+\mathrm{H}_{2} \mathrm{O} & \rightarrow & \mathrm{DMSO}_{2}+\mathrm{Ce}(\mathrm{III})+2 \mathrm{H}^{+}\end{array}$

\section{Catalyzed path}

$$
\begin{aligned}
& \mathrm{DMSO}+\mathrm{Ag}(\mathrm{I}) \quad \rightleftharpoons \quad \text { (adduct) }^{+} \text {fast step } \\
& (\text { adduct })^{+}+\mathrm{Ce}(\mathrm{IV}) \rightarrow(\text { adduct })^{2+}+\mathrm{Ce}(\mathrm{III}) \quad \text { slow step } \\
& \text { (adduct) }^{2+} \rightarrow \mathrm{DMSO}^{\circ+} \\
& \mathrm{DMSO}^{\circ+}+\mathrm{Ce}(\mathrm{IV})+\mathrm{H}_{2} \mathrm{O} \rightarrow \mathrm{DMSO}_{2}+\mathrm{Ce}(\mathrm{III})+2 \mathrm{H}^{+} \\
& \text {Scheme } 9
\end{aligned}
$$

In these reactions there is no change in concentration of $\mathrm{Ag}(\mathrm{I})$ at the end of the reaction which was confirmed by chromate titration.

\section{CONCLUSION}

From the above examples it was very clear that the process of cooxidation is different from catalysis. The essential condition for cooxidation to occur is one of the two substrates having two

\section{Only catalyzed reactions}

There were some instances in literature those are only catalyzed reactions. In the absence of the catalyst even under boiling conditions there was no reaction. One of such reactions was kinetics of $\mathrm{Ag}(\mathrm{I})$ catalyzed oxidative decarboxylation of some organic acids by $\mathrm{Ce}(\mathrm{IV})$ in $\mathrm{H}_{2} \mathrm{SO}_{4}$ medium ${ }^{32}$. The reaction sequence taking acetic acid $(\mathrm{HA})$ as an example is shown in scheme 10.

$$
\begin{aligned}
\mathrm{HA}+\mathrm{Ag}(\mathrm{I}) & \rightleftharpoons{\text { (adduct })^{+}} \\
\left({\text {adduct })^{+}+\mathrm{Ce}(\mathrm{IV})} \rightarrow{\text { (adduct })^{2+}+\mathrm{Ce}(\mathrm{III})}\right. & \text { Scheme } 10
\end{aligned}
$$

The rest of the steps are similar to the steps 6 and 7 of the scheme 7 .

functional groups must be a good chelating agent with the metal ion oxidant. And in the catalytic reaction a catalyst in micro molar $(\mathrm{mM})$ quantities must be able to catalyze the reaction with lower activation energies compared to the un-catalyzed reaction.

\section{REFERENCES}

1. Co-oxidation of isopropyl alcohol and oxalic acid by chromic acid: a one-step three electron oxidation. Fariza Hasan and Jan Rocek, J. Am. Chem. Soc., 1972, 94, 3181.

2. Three-electron oxidations. II. The chromium (VI) oxidation of oxalic acid. Fariza Hasan and Jan Ro'ek. J. Am. Chem. Soc., 1972, 94, 9073.

3. Three-electron oxidations. III. The chromium (V) oxidation step. Fariza Hasan and Jan Rocek. J. Am. Chem. Soc., 1972, 94, 8946.

4. Three-electron oxidations. IV. The chromic acid cooxidation of tertiary hydroxy acids and alcohols. Fariza Hasan and Jan Rocek, J. Am. Chem. Soc., 1973, 95, 5421.

5. Three-electron oxidation. V. The rapid reaction of chromic acid with two component substrate systems. Fariza Hasan and Jan Ro'ek. J. Org. Chem., 1973, 38, 3812.

6. Three-electron oxidation. VI. Chromic acid cooxidation of cyclobutanol and oxalic acid. The chromium (V) oxidation of cyclobutanol. Fariza Hasan and Jan Ro'ek, J. Am. Chem. Soc., 1974, 96, 534.

7. Three-electron oxidations. VII. The pre- steady-state phase of the chromic acid oxidation of oxalic acid. Fariza Hasan and Jan Rocek, J. Org. Chem., 1974, 39, 2612.

8. Three-electron oxidations. VIII. Direct evidence for the synchronous character of three-electron oxidations. F. Hasan and J. Ro'ek. J. Am. Chem. Soc., 1974, 96(70) 6802.

9. Three-electron oxidations. IX. Chromic acid oxidation of glycolic acid. Fariza Hasan and Jan Ro'ek. J. Am. Chem. Soc., 1975, 97(71). 1444.

10. Three-electron oxidations. X. Cooxidation of isopropyl alcohol and glycolic acid. F. Hasan and J. Ro'ek. J. Am. Chem. Soc., 1975, 97, 3762.

11. Three-electron oxidations. XI. Chromium(V) oxidation of alcohols. Fariza Hasan and Jan Ro'ek. J. Am. Chem. Soc., 1976, 98, 6574.

12. Three-electron oxidations. XII. Chromium(V) formation in the chromic acid oxidation of 2hydroxy-2-methylbutyric acid. Miroslav Krumpolc and Jan Ro'ek. J. Am. Chem. Soc., 1977, 99, 137.

13. Three-election oxidations. XIII. Intramolecular cooxidation of 2,7- 
dihydroxyheptanoic acid. Structure of the transition state in the chromium(VI) oxidation of alcohols. K.G. Srinivasan and J. Roèek. J. Am. Chem. Soc., 1978, 100, 2789.

14. Three-electron oxidations. XIV. Carbon-13 isotope effect in the three-electron cooxidation of isopropyl alcohol and oxalic acid. S. Ramesh, J. Roèek, and D. A. Schoeller. J. Phys. Chem., 1978, 82, 2751.

15. Three-electron oxidations. XV. Chromic acid oxidation of mandelic acid. Dominic Ip and Jan Rocek. J. Org. Chem., 1979, 44, 312.

16. Three-electron oxidations. XVI. Chromic acid oxidation of mandelic acid. Dominic Ip and Jan Rocek. J. Am. Chem. Soc., 1979, 101, 6311.

17. Three-electron oxidations. XVII. The chromium(VI) and chromium(V) steps in the chromic acid cooxidation of 2-hydroxy-2methylbutyric acid and 2-propanol. S. N. Manapatro, M. Krumpolc and J. Rocek, J. Am. Chem. Soc., 1980, 102, 3799.

18. Three-electron oxidations. XVIII. Carbon-13 and deuterium isotope effects in the cooxidation of 2hydroxy-2-methylbutyric acid and 2-propanol. evidence for a two-step mechanism. S. Ramesh, S. N. Manapatro, J. H. Lin and J. Roèek, J. Am. Chem. Soc., 1981, 103, 5172.

19. Kinetics and mechanism of oxidation of some aliphatic esters by chromic acid in the presence and absence of oxalic acid in acetic acid-water medium. P. Musala Reddy, V. Jagannadham, B. Sethuram and $T$. Navaneeth Rao, Indian J. Chem., Sect. A., 1982, 21, 483.

20. Co-oxidation of isopropanol and lactic acid in the presence of chromic acid: a case of three-electron oxidation study. V. Jagannadham, M. Anand Rao, B. Sethuram and T. Navaneeth Rao. Oxid. Commun., 1986/1985, 8, 31.

21. Three-electron oxidation: chromic acid oxidation of a mixed substrate system of an alcohol and aldehyde. P. Musala Reddy, V. Jagannadham, B. Sethuram and $\mathrm{T}$. Navaneeth Rao, React. Kinet. Catal. Lett. 1982, 19, 243.

22. Kinetics and mechanism of oxidation of glycerol by chromic acid in sulfuric acid medium. P. Musala Reddy, V. Jagannadham, B. Sethuram and T. Navaneeth Rao, Indian J. Chem., Sect. A 1982, 21, 608.
23. A rapid reaction of $\mathrm{Mn}(\mathrm{VII})$ with two component substrate systems containing 2propanol and some bi-functional compounds: a kinetic study. M. Anand Rao, B. Sethuram, T. Navaneeth Rao, and V. Jagannadham, Oxid. Commun., 1986, 9, 247.

24. The oxidation of organic substances by compounds of tervalent manganese: Oxidation of mandelic acid, ethylene glycol, glycerol and D-mannitol by the pyrophosphate complex of manganese(III) and by manganese(III) sulphate, J. Barek. .A. Berka. I. Procházková. Talanta, 1974, 21, 157.

25. 7 things one may not know about catalysis, Louise Lerner, Argonne National Laboratory, and also see the links: http://www.j agranjosh.com/general-knowledge/what-iscatalysis-1457685546-1, and https://www. google.co.in/?gfe_ rd=cr\&ei=dAziVISUF sPM8geU 7oDoCA\&g ws_rd=ssl\#q= what + is+catalysis.,2011.

26. (a) Oxidations with chromium(VI) oxide. VII. The mechanism of oxidation of secondary alcohols, J. Rocek and J. Krupicka, Collect. Czech. Chem. Commun. 1958, 23, 2068. (b) Chromic acid oxidation of iso-propyl alcohol, Kenneth B. Wiberg, and Hans Schaefer, J. Am. Chem. Soc., 1969, 91, 933-936.

27. Chromic acid oxidation of oxalic acid: kinetic investigation of the uncatalysed oxidation of oxalic acid by chromic acid, G.V. Bakore and C. L. Jain, Journal of Inorganic and Nuclear Chemistry, 1969, 31, 805-810.

28. The chromic acid oxidation of oxalic acid: Evidence of chromium(IV) oxidation, F. Hasan and J. Rocek, Tetrahedron, 1974, 30, 21-24.

29. The oxidation of isopropyl alcohol with potassium permanganate, William Lloyd Evans, Lily Bell Sefton, J. Am. Chem. Soc., 1922, 44 (10), 2271-2276

30. Theodore E. Friedemann and Arthur I. Kendall, The determination of lactic acid, J. Biol. Chem. 1929, 82, 23-43.

31. $\mathrm{Ag}(\mathrm{I})$ catalyzed and un-catalyzed oxidation of dimethyl sulfoxide by $\mathrm{Ce}(\mathrm{IV})$ in nitric acid medium: A kinetic study, S. Venkateswar Rao and V. Jagannadham, React. Kinet., Catal., Lett., 1985, 27, 239.

32. Kinetics of $\mathrm{Ag}(\mathrm{I})$ catalyzed oxidative decarboxylation of some organic acids by $\mathrm{Ce}^{4+}$ in $\mathrm{H}_{2} \mathrm{SO}_{4}$ medium, M. Adinarayana, B. Sethuram and T. Navaneeth Rao, Current Science, 1975, 44 581-583. 